\title{
Professional Attitudes towards Mental Illness: Testing the Contact Hypothesis among Nurses of Ethnic Anglo and Chinese Australian Backgrounds
}

\author{
Tan Kan Ku1, Michael Ha², Uthit Siriwan ${ }^{3}$ \\ ${ }^{1}$ Nurse Educator, Victoria University Institute of Technology (VUIT), St. Albans Campus, Melbourne, Australia \\ ${ }^{2}$ Department of Mathematical Sciences, Xi'an Jiaotong-Liverpool University, Suzhou, China \\ ${ }^{3}$ Faculty of Business, Charisma University, Turk and Caicos Islands, UK \\ Email: tankanku@gmail.com, Tan.Ku@vu.edu.au, Michael.Ha@xjtlu.edu.cn, \\ U.Siriwan@charismauniversity.info
}

Received 9 June 2015; accepted 7 July 2015; published 14 July 2015

\begin{abstract}
Background: Professional attitudes towards mental illness may be related to contact level and ethnicity has not been thoroughly examined in health care practices although this has been stated to be the case. The literature indicated contact through a course of study or clinical placement usually improved attitudes of health workers towards mental illness. Purpose: The aim of this study was to develop a measure (labelled as Contact with Psychiatric Patient (CPP) Scale to compare attitudes towards mental illness between General and Psychiatric nurses of Anglo and Chinese Australian backgrounds. Methods: A cross-sectional survey was presented to $\mathbf{7 6}$ general and 132 psychiatric nurses. Twenty one questions of the nurse-patient contact level were asked. Principal component analyses (with oblique rotation) were used to identify underlying dimensionality in the correlations of items. Subscale score variations were analysed across nurse type and ethnicity to examine the discriminant validity of the subscales. Results: Principal component analysis revealed four dimensions accounting for 67.3 percent of the variation within items. Four "conceptual" factors were derived. These were labelled Contact-Through-Work Situation, Patient Helps Nurse, Relative to Mental Illness and External Socialisation with Patient. Developed as subscales, reliability analysis indicated moderate to high internal consistency with respective alpha coefficients of $0.94,0.73,0.55$ and 0.81 . There was significant variation in scale scores according to nurse type (psychiatric versus general) and ethnicity (Anglo versus Chinese). Psychiatric nurses endorsed a higher level of contact level than general nurses. Conclusion: Psychometric evaluation of the CPP suggests that it is a reliable instrument for measuring four key dimensions of the nurse-patient contact relationship and it enables the study of this relationship cross-culturally in large samples.
\end{abstract}

\section{Keywords}

Mental Illness, Ethnicity, Psychiatric, Cross-Culture, Attitude 


\section{Introduction}

Social researchers and advocates have agreed that one way of challenging the stigma of mental illness is by attitudes studies. Contact has proven to be the best strategy for reducing negative attitudes towards mental illness when compared with knowledge and protest (Corrigan et al., 2001) [1]. Contact can be interpersonal contact with people with a mental illness either retrospectively or prospectively (Couture and Penn, 2003) [2].

Despite a large number of studies in the literature associating contact (independent variable) with mental illness attitudes (as a dependent variable) and examining clinical placement effect (as a form of short term contact) on medical students (Reddy et al., 2005) [3] (Altindag et al., 2006) [4] and undergraduate nurses' attitudes (Callaghan et al., 1997) [5] (Evagelou et al., 2005) [6] (Madianos et al., 2005) [7], few researchers have examined the effect of contact using samples of qualified nurses of different ethnic backgrounds in Australia. Our study aims to fill this gap.

\subsection{Literature Review}

The effect of a course of psychiatric secondment and contact on attitudes towards mental illness was investigated among Hong Kong Chinese student nurses $(\mathrm{N}=92)$ by Callaghan et al. [5]. One of two groups of students undertook a psychiatric secondment. Findings did not support the contact hypothesis as both groups (control and experimental) in this Hong Kong study expressing general positive attitudes towards mental illness (i.e., a ceiling effect).

Evagelou et al. ( 2005) [6] and Madianos et al. (2005) [7] published similar work in Greece using the Opinion of Mental Illness (OMI) by Cohen and Struening (1965) [8] (with subscales of Social Discrimination, Social Restriction, Social Care, Social Integration, and Etiology) to measure nurses' attitudes in a pre-post design. In Madianos et al.'s [7] study, a sample of 92 undergraduate students in the School of Nursing of the University of Athens participated in a study for assessment of attitudes before and after attending lectures (40 hours) and clinical placement (90 hours). Attitudes were examined before the first lecture (Time 1) and reassessed with vignette presentations after completion of the psychiatric practicum, six months afterwards (Time 2). By Time 2, results showed that students were less authoritarian and scored lower social discrimination and restrictiveness. Students were also more willing to accept a mentally ill person as a neighbour or an employee. Furthermore, students reported more positive attitudes towards community integration of the mentally ill, and by Time 2 , they had a better knowledge of causes of mental illness.

In a similar design, Evagelou et al. (2005) [6] assessed 137 undergraduate students' attitudes before (Time 1) and after completion (Time 2) of 105 hours course of psychiatric nursing at a technological nursing school in Athens, Greece. Twenty five students had a relative with mental illness. Results indicated that students endorsed less social restriction and were more positive towards social integration of the mentally ill after the course. Male students endorsed a more positive attitude toward social care after the course. Female students reported more tolerance (i.e., lower endorsement of social restriction of the mentally ill) and were more positive toward social integration of patients with mental illness than male students. The authors suggested that the gender difference might be due to the fact that females were more emotion-focused and humanistic than males and this was reflected in their attitudes towards the mental illness. Those students who were single having a relation to mental illness were raised in an urban place, or those having no occupation reported more acceptance of social integration of the mental illness.

The association among attitudes, clinical exposure and career choice was examined by Surgenor, Dunn and Horn (2005) [9] in Christchurch, New Zealand. One hundred and sixty-four nursing students from a tertiary training institution participated in a cross-sectional survey. Results indicated that third year students who had been exposed to theoretical knowledge and clinical experience in psychiatry reported more positive attitudes towards mental illness than first year students who intended to specialize in paediatrics and expressed the most negative attitudes towards psychiatric nursing. Nevertheless, psychiatry remained as the least popular specialty after registration as a qualified nurse. Those with more positive attitudes towards mental illness were more likely to intend on a career in psychiatry. Students who had multiple forms of exposure reported more positive attitudes than those whose contact was only via work. Age and gender were not associated with attitudes.

\subsection{Hypotheses}

1) When comparing with General nurses, Psychiatric nurses by virtue of the specialty choice and greater expe- 
rience with mentally ill patients will endorse greater level of contact with mentally ill people;

2) Ethnicity of the nurses will influence contact level with mentally ill people, i.e., Anglo nurses will endorse greater level of contact than Chinese nurses, possibly due to Chinese nurses being immigrants in Australia which inevitably results in lesser time in encountering people with mental illness.

\section{Methods}

\subsection{Subjects}

Two hundred and eight (208) nurses participated in this study. They were selected for the purpose of a broader study on the effect of cultural factors in nursing practice within the psychiatric and general settings. As a result the sample was composed of 83 Anglo-Australian and49 Chinese-Australian psychiatric nurses and, 41 Anglo-Australian and 35 Chinese-Australian general nurses. One hundred and forty-eight (148) nurses were females and 60 were males. The mean age of the sample was 44.8 years (s.d. $=9.6)$, ranging from 21 to 65 years of age. For the overall sample the mean number of working years in general setting was $11.2($ s.d. $=12.0)$ and in psychiatric setting was 9.8 (s.d. $=11.0)$ respectively. Table 1 shows the sample demographics and Table 2 shows the period of immigrant nurses to Australia.

Table 1. Characteristics of the sample $(\mathrm{N}=208)$.

\begin{tabular}{|c|c|c|c|c|c|}
\hline & Chinese & Chinese & Anglo & Anglo & $\chi^{2} / \mathrm{F}$ value \\
\hline & Psychiatry & General & Psychiatry & General & \\
\hline & $(\mathrm{n}=49)$ & $(\mathrm{n}=35)$ & $(\mathrm{n}=83)$ & $\underline{(n=41)}$ & \\
\hline $\mathbf{A g e}^{\#}$ (mean, s.d.) & $50.3(5.8)$ & $47.9(6.0)$ & $43.1(9.6)$ & $39.5(11.6) 13.2^{* * *}$ & $\mathrm{~F}(1,197)$ \\
\hline Sex (male/female) & $25 / 24$ & $1 / 34$ & $31 / 52$ & $3 / 38$ & $35.4^{* * *}$ \\
\hline \multirow[t]{2}{*}{ Years in Mental Health Nursing } & (Mean, s.d.) & & & & $\mathrm{F}(1,200)$ \\
\hline & $19.5(10.4)$ & $0.03(0.1)$ & $12.9(9.2)$ & $0.07(0.2)$ & $68.8^{* * *}$ \\
\hline \multirow[t]{2}{*}{ Years in General Nursing } & (Mean, s.d.) & & & & $\mathrm{F}(1,204)$ \\
\hline & $\underline{7.7(10.5)}$ & $\underline{23.1(9.0)}$ & $\underline{6.1(9.3)}$ & $\underline{15.6(12.5)}$ & $\underline{27.3^{* * *}}$ \\
\hline
\end{tabular}

${ }^{\sharp} 7$ missing cases are excluded from the analysis in age. ${ }^{* * *} \mathrm{p}<0.001$. For all $\chi^{2}$ analyses, $\mathrm{df}=3$.

Table 2. Number of immigrant nurses and period of arrival in Australia.

\begin{tabular}{ccc}
\hline Period of Arrival & Chinese Nurses & Anglo Nurses \\
\hline $1950-1959$ & $\underline{\text { Frequency }(\%)^{1}}$ & $\underline{\text { Frequency }(\%)^{2}}$ \\
$1960-1969$ & $0(0.0 \%)$ & $4(12.1 \%)$ \\
$1970-1979$ & $2(2.4 \%)$ & $6(18.2 \%)$ \\
$1980-1989$ & $29(34.5 \%)$ & $7(21.1 \%)$ \\
$1990-1999$ & $46(54.8 \%)$ & $9(27.3 \%)$ \\
$\underline{2000-2002}$ & $4(7.1 \%)$ & $4(18.2 \%)$ \\
\hline
\end{tabular}

${ }^{12}$ Chinese nurses were born in Australia; ${ }^{2} 111$ Anglo nurses were born in Australia. 


\subsection{Item Development of the CPP}

A survey with twenty-one (21) questions was constructed (available upon request). Most of the source of 'contact' were drawn from the first author, Ku's experience as a psychiatric nurse asking the nurses to identify different types and level of contact through their encounters with patients, relatives, friends or colleagues who have a mental illness. It explored nurses' contact with people with mental illness, such as having "a long talk", "brief conversation", "home visit", "going to the cinema" among other items, which could be in social situations. Contact events could also be adverse events such as having to apply "physical restraint" and "seclusion" which could occur in situation when working in a high dependency ward in an acute mental health setting. A score of "1" represented never had contact, " 2 " for a few times of contact and "3" for many times via each particular means of contact.

\subsection{Procedure}

Recruitment of participants was achieved through a snowballing technique. After ethics approval for the study was granted by the University of Melbourne Human Research Ethics Committee (HERC No. 020030), an initial pool of general and psychiatric nurses $(n=20)$ of Chinese-Australian and Anglo-Australian backgrounds working in various institutions were identified and asked to participate in the study. Nurses in the initial pool known to the first author $(\mathrm{Ku})$ were asked to talk with potential participants and ask permission for the first author to approach them to introduce the study formally. Those nurses interested in participating were asked to meet with the first author for the purpose of further explanation of the nature, purpose and procedure of the study. All participants signed a written consent form to anonymous participation. Data were collected in the latter part of 2002 and early 2003. The CPP items followed after demographic items for the nurses to score their responses.

\subsection{Response Rate}

Three hundred and forty (347) surveys were disturbed. Two hundred and eight nurses out of 331 relevant participants returned the survey giving a $63 \%$ response rate $(208 / 331 \times 100)$.

\subsection{Statistical Analysis}

Principal component analyses were used to identify common dimensions underlying the variation of the item scores of the CPP. Cronbach's alpha coefficients were calculated to estimate the internal reliability of the derived CPP subscales. Two-way analysis of variance was used to examine the discriminant validity of the subscales. All analyses were conducted using the Statistical Package for the Social Sciences (SPSS Version 12).

\section{Results}

\subsection{Dimensions of the CPP}

A number of preliminary principal components analyses were con ducted to explore the dimensionality of the CPP. Factor solution was aided by the use of the Scree test and in consideration of the Kaiser criterion of eigenvalues greater than or equal to one. Four factors were identified and one item removed from the final solution due to its low correlation with any of the four factors. The four dimensional solution accounted for 67.3 percent of the variance in items.

Treating each of the factors as scales, reliability analysis revealed relatively good internal consistency among scale items. Alpha coefficients are summarised in Table 3.

The first factor was labelled Contact-Through-Work Situation (CWS) as items included situations such as "secluding a person with a mental illness", use of "physical restraint" among other situations and activities that can be considered to occur in patient contact within an acute psychiatric environment. Factor 2 identifies items relating to close contact where the nurse is receiving support or allows interaction in relation to the nurse's private life from a person with a mental illness. Two items suggest that the "patient" may be a colleague or friend. This factor was labelled Patient Helps Nurse (PHN). The third factor, comprised of only two items, indicates whether the nurse has a relative with a mental illness, labelled Relative with Mental Illness (RMI). The last factor, composed of three items, relates to contact with a person with a mental illness outside of the work environment reflecting some level of socialization, labelled External Socialization with Patient (ESP). 
Table 3. Pattern matrix for the CPP scale.

\begin{tabular}{|c|c|c|c|c|c|}
\hline Item & $\mathrm{F} 1$ & $\mathrm{~F} 2$ & F3 & $\mathrm{F} 4$ & Communality \\
\hline Q2 discuss their life & 0.91 & 0.15 & 0.03 & -0.14 & 0.84 \\
\hline Q1 long talk & 0.90 & 0.16 & 0.07 & -0.17 & 0.81 \\
\hline Q5 brief conversations & 0.88 & 0.15 & 0.10 & -0.13 & 0.80 \\
\hline Q8 try to help them & 0.81 & 0.09 & 0.11 & 0.04 & 0.76 \\
\hline Q19 seclude a patient & 0.79 & -0.12 & -0.04 & 0.24 & 0.80 \\
\hline Q4 high dependency & 0.79 & -0.91 & 0.02 & 0.20 & 0.75 \\
\hline Q16 verbal abuse & 0.78 & -0.10 & 0.05 & 0.15 & 0.70 \\
\hline Q7 physical restraint & 0.77 & -0.08 & -0.04 & 0.23 & 0.75 \\
\hline Q13 physical violence & 0.59 & -0.12 & -0.05 & 0.24 & 0.49 \\
\hline Q6 meal with & 0.55 & 0.35 & -0.02 & 0.13 & 0.61 \\
\hline Q9 they help me & -03 & 0.78 & -0.15 & 0.06 & 0.60 \\
\hline Q3 discuss my life & 0.15 & 0.76 & -0.10 & -0.09 & 0.63 \\
\hline Q17 they visit me & -0.32 & 0.53 & 0.30 & 0.38 & 0.58 \\
\hline Q10 colleague suffers & 0.41 & 0.52 & -0.08 & 0.10 & 0.59 \\
\hline Q21 friend suffers & 0.11 & 0.49 & 0.30 & -0.05 & 0.43 \\
\hline Q20 distant relative suffers & 0.10 & -0.12 & 0.82 & -0.12 & 0.65 \\
\hline Q18 close relative suffers & 0.01 & 0.00 & 0.80 & 0.13 & 0.69 \\
\hline Q15 taken them cinema & 0.22 & 0.02 & 0.02 & 0.67 & 0.62 \\
\hline Q14 driven someone & 0.44 & 0.00 & -0.06 & 0.60 & 0.76 \\
\hline Q12 visit someone & 0.29 & 0.17 & 0.03 & 0.58 & 0.65 \\
\hline Percent variance & 44.8 & 10.9 & 6.5 & 5.2 & Total variance $=67.3$ \\
\hline Cronbach's alpha & 0.94 & 0.73 & 0.55 & 0.81 & \\
\hline
\end{tabular}

F1: CWS; F2: PHN; F3: RMI; F4: ESP.

\subsubsection{Group Contrasts of Contact with Psychiatric Patients Scores}

Two-way analysis of variance was used to examine differences in the contact scales between nurse type and ethnic groups ( 2 nurse types $\times 2$ ethnic groups). The first analysis focused on examining Contact-ThroughWork Situation (CWS) scores. The main effect of nurse type was significant $(F(1,204)=571.39, p<0.001)$ as was the main effect of ethnicity $(\mathrm{F}(1,204)=14.11, \mathrm{p}<0.001)$. Inspection of the means indicated that psychiatric nurses (mean $=2.73$, s.d. $=0.26$ ), as might be expected, endorsed higher contact levels than general nurses $($ mean $=1.69$, s.d. $=0.39)$ on this variable. Additionally, Chinese nurses $($ mean $=2.22$, s.d. $=0.66)$ reported lower overall contact with psychiatric patients according to this scale than Anglo nurses (mean $=2.44$, s.d. $=$ $0.52)$. Furthermore, the interaction term was significant $(\mathrm{F}(1,204)=11.86, \mathrm{p}<0.01)$ indicating that contact level differed across nurse types and ethnic groups. Inspection of the plot of the interaction indicated that while there were little differences between psychiatric nurses from Chinese and Anglo backgrounds in their responses (despite their former greater mental health work experience shown earlier), Chinese general nurses reported lower contact than Anglo general nurses.

The second analysis focused on examining Patient Helps Nurse (PHN) scores. The main effect of nurse type was significant $(\mathrm{F}(1,204)=25.56, \mathrm{p}<0.001)$ as was the main effect of ethnicity $(\mathrm{F}(1,204)=5.06, \mathrm{p}<0.05)$. The interaction effect was not significant $(\mathrm{F}(1,204) \leq 1)$. Inspection of the means indicated that psychiatric nurses $($ mean $=1.63$, s.d. $=0.36$ ) endorsed more support to themselves from someone with mental illness than 
general nurses $($ mean $=1.35$, s.d. $=0.37)$. Anglo nurses $($ mean $=1.59$, s.d. $=0.40)$ generally reported higher level of such contact than Chinese nurses (mean $=1.45$, s.d. $=0.36)$.

The third analysis examined Relative with Mental Illness (RMI) scores. The main effect of ethnicity was significant $(\mathrm{F}(1,204)=4.94, \mathrm{p}<0.05)$. The main effect of nurse type was not significant $(\mathrm{F}(1,204)=1.72, \mathrm{p}>$ $0.05)$. Anglo nurses (mean $=1.50$, s.d. $=.47)$ reported higher overall contact with psychiatric patients according to this scale than Chinese nurses $($ mean $=1.34$, s.d. $=0.44)$. The interaction effect was not significant $(F(1,204)$ $\leq 1)$.

In relation to examining the fourth factor, External Socialisation with Patient (ESP) scores, the main effect of nurse type was significant $(\mathrm{F}(1,204)=154.09, \mathrm{p}<0.001)$ but the main effect of ethnicity was not $(\mathrm{F}(1,204) \leq$ $1)$. The interaction term was not significant $(F(1,204) \leq 1)$. Inspection of the means indicated that psychiatric nurses $($ mean $=2.18$, s.d. $=0.54)$ had higher such contact levels than general nurses $($ mean $=1.29$, s.d. $=0.38)$.

\section{Discussion}

\subsection{Outcome in Relation to Hypothesis Testing}

The study provided support for hypothesizing that when compared with general nurses, psychiatric nurses would endorse greater contact with mentally ill people (Hypothesis 1).Greater contact was observed on factors measuring work related contact, CWS and PHN, but also on external socialisation (ESP), although no difference was observed on contact due to access to a relative having a mental illness (RMI).

Hypothesis 2 was supported as results indicated that Chinese nurses endorsed lesser contact with mentally ill people than Anglo nurses.

\subsection{Implications for Mental Health Nursing Practice}

Contact is considered a major factor in influencing attitudes towards people with a mental illness. The effect of contact can better be understood by comparing the varying degrees of contact. Mental health professionals have substantial contact with those with a diagnosed mental disorder, but the nature of their contact may lead to different attitudes, for example, they may find it more hectic and burdensome working in an admission ward caring for patients with acute psychotic episode, needing constant assessment and observation due to the acute states of patients, potential for suicide, aggressiveness and unpredictable behaviour. It is usually more relaxed working in a rehabilitation unit where patients do not need constant supervision.

In our study, we measured only retrospective contacts via work situation that is day-to-day interaction when a nurse engaged in procedures such as 'putting a patient in seclusion' or when a nurse took a patient out for a drive. Outside the work environment, contact was measured through the nurse having a relative with a mental illness or externally in social situation. Results suggest that psychiatric than general nurses have experienced more formal and informal contact with people having a mental illness and they appear to have had greater experience of relationships where social competency is implicated on the part of the mentally ill. That is, they endorsed more support received from people with mental illness compared with general nurses. Similarly Anglo nurses appear to have had greater informal and 'competent-patient' exposure than Chinese nurses. It was originally thought that positive and negative encounters may also be a useful distinction in view of Yamamoto et al.'s (1996) [10] delineation of unpleasant contact), however, in our analysis positive and negative contact experience seem to be related to the same factor (Factor 1, Contact via Work Situation).

In Callaghan et al.'s study (1997) [5], more of the experimental group had lived with a mentally ill relative and undergoing a psychiatric secondment, so their prior social contact might have already led to their positive attitudes before any training. However, it is unclear why the control group matched them on positive attitudes with the experimental group, unless the results can be taken to mean that prior social contact with the mentally ill has no material influence on attitudes.

While years of mental health nursing was used to define nurse type, and asmight be expected, to some extent, because psychiatric nurses had more experience (by definition of the groups in this study) of people with mental illness and therefore they may feel safe in their knowledge and skills for interacting with the mentally ill, because they have a personal interest in caring for the mentally ill, as shown by their choice of specialty, or, because they have experienced people with mental illness in a wider variety of settings and roles (as measured by the CPP Scale). This implies that contact, experience and choice of specialty in nursing may be significant de- 
terminants of attitudes towards mental illness amongst nurses. There have not been any other comparative studies between general and psychiatric nurses to be able to suggest that these findings generalize to other settings. The few studies of other disciplines show mixed results (Kua et al., 2000) [11] (Caldwell \& Jorm, 2001) [12].

In Caldwell and Jorm's (2001) [12] study, for example, psychiatric nurses were more optimistic about outcomes for the mentally ill than other professionals. However, these professional groups also included psychiatrists and clinical psychologists who may be similar to the psychiatric nurses in choosing mental health as their specialty. Kua et al. (2000) [11] on the other hand compared general medical practitioners and psychiatrists. Findings suggested more optimism amongst psychiatrists who endorsed better outcomes for the mentally ill, consistent in principle with present findings for nurses. However, clearly, these previous studies measured constructs that could be related to stigmatizing attitudes but did not directly measure such attitudes. On the other hand, studies of mental health training of student nurses) by Surgenor, Dunn and Horn (2005) [9], Evagelou et al. (2005) [6] and Madianos et al. (2005) [7] have shown some reductions in negative attitudes towards the mentally ill. Such results are consistent with present findings, but are more specifically indicative of the effects of training exposure and short term experience on attitudes rather than effects due to choice of specialty. However, training effects have been inconsistent and are probably due to specific factors (Reed and Fitzgerald, 2005) [13], and may not last (Baxter et al., 2001) [14] as had been documented in the literature.

\section{Limitation of Study}

Although demographic characteristics (age and gender of the sample conform to the general trend in the Victorian nursing workforce (Nurses Board of Victoria, 2005) [15], the results may not be representative of the nursing population in Victoria. The sample consisted only of nurses from Metropolitan Melbourne who were self-selected to participate, which might imply interest in mental health issues that may not be shared by the wider nursing community (especially given the evidence regarding specialty choice in the literature). This is a particularly important consideration given the small sample involved in the study constituting only a small fraction of the overall nursing community. Furthermore, the study measured retrospective attitude which could be the impact of circumstances (via work or having a relative with mental illness, etc) encountered by the nurse during his/her contact with mental illness, hence, we should be cautious not to generalise our findings to include all qualified nurses' attitudes.

\section{Recommendation and Future Research}

\subsection{Sample}

Given the paucity of Australian research on professionals' attitudes towards mental illness, future research may be directed to include other health professionals (doctors, psychologists, occupational therapists, social workers) working in different mental health settings in order to compare their views as a result of their work, personality style and interactional style.

\subsection{Methodology}

The quantitative design of the present study is a cross-sectional survey which is researcher-controlled and aiming at obtaining generalisation of findings. Thus, in future research, it is essential to incorporate qualitative data in attitudinal study to ascertain the lived experience of participants. This method of triangulating across paradigms using multiple sources (survey and personal interviews) in a single study aims to offset the limitations of each approach by counterbalance biases (entail research control in quantitative survey) and to achieve credibility (one dimension of trustworthiness in qualitative method).

What has been of light is that among the 208 nurses, Chinese nurses reported less contact with mental illness than Anglo nurses, contact level was better placed as a mediator between stigmatising attitudes and differential clinical approaches among the 84 Chinese immigrant nurses in Australia using structural equation modelling, when Chinese cultural values, as a latent variable, was analysed using path analyses (2015) [16]. Subsequently, the first author $(\mathrm{Ku})$ has embarked on a PhD study which comprised both quantitative and qualitative design by not involving Anglo-culture participants, but to further examine what specifically constitutes stigma of mental illness in the wider Chinese community, focusing on uncovering socio-cultural factors influencing Chinese people's attitudes towards depression and schizophrenia, two commonly known mental disorders (2015) [17]. 


\section{Conclusion}

Despite some of the methodological limitations in the present study, this study has generated some original data otherwise unavailable previously in Australia. Those working with, suffering from or having a relation to mental illness in Australia can draw some light from our findings. In view of Psychometric evaluation of the CPP, it is a reliable instrument for measuring four key dimensions of the nurse-patient contact relationship cross-culturally in mapping attitudes and professional practice.

\section{Authors' Contributions}

The first author prepared the first draft of the paper and the second and the third authors verified statistical results and edited the paper. All authors have approved the final version of the manuscript.

\section{Acknowledgements}

The authors would like to thank Victoria University Institute of Technology and Xi'an Jiaotong-Liverpool University for supporting and sponsoring the presentation of this paper.

\section{References}

[1] Corrigan, P.W., River, L.P., Lundin, R.K., Penn, D.L., Uphoff-Wasowski, K., Campion, J., Mathisen, J., Gagnon, C., Bergman, M., Goldstein, H. and Kubiak, M.A. (2001) Three Strategies for Changing Attributions about Severe Mental Illness. Schizophrenia Bulletin, 27, 187-195. http://dx.doi.org/10.1093/oxfordjournals.schbul.a006865

[2] Couture, S.M. and Penn, D.L. (2003) Interpersonal Contact and the Stigma of Mental Illness: A Review of the Literature. Journal of Mental Health, 12, 291-305. http://dx.doi.org/10.1080/09638231000118276

[3] Reddy, J.P., Tan, S.M.K., Azmi, M.T., Shaharom, M.H., Rosdinom, R., Maniam, T., Ruzanna, Z.Z. and Minas, I.H. (2005) The Effect of a Clinical Posting in Psychiatry on the Attitudes of Medical Students towards Psychiatry and Mental Illness in a Malaysian Medical School. Annals, Academy of Medicine, Singapore, 34, 505-510.

[4] Altindag, A., Yanik, M., Ucok, A., Alptekin, K. and Ozkan, M. (2006) Effects of an Antistigma Program on Medical Students' Attitudes towards People with Schizophrenia. Psychiatry and Clinical Neuroscience, 60, 283-288. http://dx.doi.org/10.1111/j.1440-1819.2006.01503.x

[5] Callaghan, P., Shan, C.S., Yu, L.S., Ching, L.W. and Lai, T. (1997) Attitudes towards Mental Illness: Testing the Contact Hypothesis among Chinese Student Nurses in Hong Kong. Journal of Advanced Nursing, 26, 33-40. http://dx.doi.org/10.1046/j.1365-2648.1997.1997026033.x

[6] Evagelou, H., Adali, E., Koukia, E., Katostaras, F., Priami, M. and Toulia, G. (2005) The Influence of Education of Nursing Students on the Formation of Attitudes toward Psychiatric Illness. ICUS and Nursing Web Journal, 1-8.

[7] Madianos, M., Priami, M., Aleviscopoulous, G., Koukia, E. and Rogakou, E. (2005) Nursing Students' Attitudes Change towards Mental Illness and Psychiatric Case Recognition after a Clerkship in Psychiatry. Issues in Mental Health Nursing, 26, 169-183. http://dx.doi.org/10.1080/01612840590901635

[8] Cohen, J.S. and Struening, E.L. (1965) Opinions about Mental Illness: Hospital Differences in Attitudes for Eight Occupational Groups. Psychological Reports, 17, 25-26. http://dx.doi.org/10.2466/pr0.1965.17.1.25

[9] Surgenor, L.J. Dunn, J. and Horn, J. (2005) Nursing Student Attitudes to Psychiatric Disorders in New Zealand. International Journal of Mental Health Nursing, 14, 103-108. http://dx.doi.org/10.1111/j.1440-0979.2005.00366.x

[10] Yamamoto, K., Randall, M., Takeda, M. and Leeamanit, W. (1996) Attitudes of Medical Students towards Persons with Mental Disorders: A Comparative Study between Japan and Thailand. Psychiatry and Clinical Neuroscience, 50, 171-180. http://dx.doi.org/10.1111/j.1440-1819.1996.tb02738.x

[11] Kua, J.H.K., Parker, G., Lee, C. and Jorm A.F. (2000) Beliefs about Outcomes for Mental Disorders: A Comparative Study of Primary Health Practitioners and Psychiatrists in Singapore. Singapore Medical Journal, 41, 542-547.

[12] Caldwell, T.M. and Jorm, A.F. (2001) Mental Health Nurses' Beliefs about Likely Outcomes for People with Schizophrenia or Depression: A Comparison with the Public and Other Health Care Professionals. Australian and New Zealand Journal of Mental Health Nursing, 10, 42-54. http://dx.doi.org/10.1046/j.1440-0979.2001.00190.x

[13] Reed, F. and Fitzgerald, L. (2005) The Mixed Attitudes of Nurse's to Caring for People with Mental Illness in a Rural General Hospital. International Journal of Mental Health Nursing, 14, 249-257. http://dx.doi.org/10.1111/j.1440-0979.2005.00389.x

[14] Baxter, H., Singh, S.P., Standen, P. and Duggan, C. (2001) The Attitudes of Tomorrow's Doctors' towards Mental Illness and Psychiatry: Changes during the Final Undergraduate Year. Medical Education, 35, 381-383. 
http://dx.doi.org/10.1046/j.1365-2923.2001.00902.x

[15] Nurses Board of Victoria (2005) Nexus, 12, 1-11. Nurse Board of Victoria, Melbourne.

[16] Ku, T.K. and Ha, M. (2015) Culture and Stigma of Mental Illness: Path Analysis Conducted with Amos in Transcultural Psychiatry in Australia. Journal of Biosciences and Medicines, 3, 15-22. http://dx.doi.org/10.4236/jbm.2015.36003

[17] Ku, T.K. and Ha, M. (2015) Development of the Attitudes towards Depression and Schizophrenia Scale (ADSS). Studies in Social Sciences and Humanities, 2, 12-20. 